\title{
A INFLUÊNCIA DOS MODOS DE VARIABILIDADE CLIMÁTICA NA TEMPERATURA EM SUPERFÍCIE NA ANTÁRTICA
}

\author{
DOUGLAS DA SILVA LINDEMANN, FLÁVIO BARBOSA JUSTINO
}

\author{
Universidade Federal de Viçosa, Departamento de Engenharia Agrícola,Viçosa, MG, Brasil \\ douglasdasilva.lindemann@gmail.com, fjustino@ufv.br
}

Recebido Agosto de 2012 - Aceito Julho de 2014

\begin{abstract}
RESUMO
Este trabalho analisa a variação sazonal da temperatura do ar à superfície $\left(\mathrm{T}_{\mathrm{ar}}\right)$ na Antártica, e sua relação com os modos de variabilidade climática El Niño-Oscilação Sul (ENOS) e Oscilação Antártica (OA). Os resultados indicam que para os 4 períodos sazonais as $\mathrm{T}_{\mathrm{ar}}$ nas estações localizadas no setor oriental da Antártica apresentaram significativas correlações negativas (nível de significância de 1\% na maioria das estações) com a OA. Todavia o único período sazonal, em que o setor continental (Amundsen-Scott, Dome C) apresentou forte correlação negativa com a OA, foi durante o inverno (significativa a 1\%). Correlações positivas foram encontradas (significâncias entre 1\% e 5\%) entre as $T_{\text {ar }}$ da Península Antártica e Shetland do Sul com a OA para os períodos de outono, inverno e primavera. Os resultados demonstram a influência que a OA exerce sobre a climatologia da $T_{a r}$ na Antártica. É importante notar um dipolo entre as regiões oriental da Antártica e da Península Antártica, no que concerne as influências da OA. O modo de variabilidade ENOS apresentou correlação negativa significativa com as três estações da Península Antártica somente durante a primavera, enquanto que para a estação Rothera, à oeste da Península, a correlação foi significativa também para o outono e inverno. A correlação entre ENOS e $\mathrm{T}_{\mathrm{ar}}$ foi positiva e significativa no setor do Mar de Ross durante a primavera, e com o setor oriental da Antártica durante o verão, com nível de significância chegando a 5\%.

Palavras Chave: Antártica; temperatura; modos de variabilidade.
\end{abstract}

\begin{abstract}
THE INFLUENCE OF THE MODES OF CLIMATE VARIABILITY IN ANTARCTIC SURFACE TEMPERATURE

This study analyzes the seasonal variation in surface air temperature $\left(\mathrm{T}_{\mathrm{ar}}\right)$ in Antarctica, and its relationship with the modes of climate variability, namely El Niño-Southern Oscillation (ENSO) and Antarctic Oscillation (AO). The results indicate that during the 4 seasonal periods the $\mathrm{T}_{\mathrm{ar}}$ in the stations located in the eastern sector of Antarctica showed significant negative correlations (at $1 \%$ significance level on most stations) with AO. However, the only seasonal period in which the continental sector (Amundsen-Scott, Dome C), showed strong negative correlation with the AO was in winter (at 1\%). Positive correlations were found (ranging from $1 \%$ to $5 \%$ ) between the Tar values at the Antarctic Peninsula and South Shetland with AO for autumn, winter and spring. These results demonstrate the influence that AO exerts on the climatology of the Tar in Antarctica. It is important to note a dipole between eastern regions of Antarctica and the Antarctic Peninsula concerning the influences of AO. The ENSO mode presented significant negative correlation with the three stations at the Antarctic Peninsula only during the spring, while for the station Rothera, at the West sector the correlation was also significant for the autumn and winter. The correlation between ENSO and Tar was positive and significant in the Ross Sea sector during the spring, and with the eastern sector of Antarctica during the summer, with significance level reaching 5\%.
\end{abstract}

Keywords: Antarctic; temperature; variability modes. 


\section{INTRODUÇÃO}

A região da Antártica é um componente ativo no sistema climático global, que é influenciado pelos modos de variabilidade climáticos globais, regionais e locais (Yuan, 2004; Yu et al., 2011). O sistema climático global é afetado por vários modos internos de variabilidade que são caracterizados pela diferença dos padrões temporais e espaciais. Nas regiões extratropicais do Hemisfério Sul (HS), o padrão dominante de variabilidade climática é o Southern Annular Mode (SAM) ou Modo Anular do Hemisfério Sul, também conhecido como Antarctic Oscillation (AAO) ou Oscilação Antártica (Carvalho et al., 2005). A Oscilação Antártica (OA) é responsável por aproximadamente $35 \%$ do total da variabilidade climática no HS (Justino e Peltier, 2008). Essencialmente a OA é uma estrutura anular com anomalias de pressão zonalmente simétricas e com sinais opostos em altas e médias latitudes. A polaridade positiva da OA está associada com anomalias negativas na temperatura do ar em superfície $\left(\mathrm{T}_{\mathrm{ar}}\right)$ e altura geopotencial sobre o continente Antártico e anomalias positivas nas latitudes médias (Marshall, 2007). Nesta fase observa-se aumento da $\mathrm{T}_{\mathrm{ar}}$ na Península Antártica e Shetland do Sul, intensificação dos ciclones sobre o Oceano Austral e dos ventos de leste em torno de $60^{\circ} \mathrm{S}$. Situações onde os meses correspondem a baixos índices de polaridade são marcadas por anomalias no sentido oposto (Justino e Peltier, 2008; Thompson e Solomon, 2002; Thompson e Wallace, 2000).

$\mathrm{Na}$ região equatorial e considerando escalas de tempo interanuais, as condições climáticas são influenciadas pelas fases do El Niño-Southern Oscillation (ENSO) ou El NiñoOscilação Sul (ENOS) (Feldstein, 2000). Hoskins e Karoly (1981) mostraram que regiões de convecção profunda próxima à região equatorial podem gerar ondas de Rossby, através da vorticidade gerada por aquecimento adiabático. Estas ondas movem-se em direção aos pólos em ambos os hemisférios e induzem teleconexão entre ENOS e o clima de médias e altas latitudes. A influência do ENOS foi descrita em estudos com base na análise dos campos de vento em $200 \mathrm{hPa}$ (Carvalho et al., 2005), de pressão em 500 hPa (Mo e White, 1985).

Estes principais modos de variabilidade climática podem influenciar as tendências de Tar na região da Antártica em determinados períodos, podendo mudar os regimes climáticos desta região. Tendências de $T_{a r}$ na Antártica podem ser determinadas usando diferentes formas de dados, incluindo observações in situ, gelo, reanálises, imagens de satélites, entre outros (King e Turner, 1997). Nos últimos anos muitos estudos examinaram as variações climáticas em toda a Antártica usando dados de estações meteorológicas (Jacka e Budd, 1991; Jacka e Budd, 1998; Raper et al., 1984). É importante ressaltar que a $T_{a r}$ registrada na Antártica está restrita a algumas estações.
A quantidade de estações disponíveis com uma boa série de dados ainda é pequena e a maioria das estações está localizada na periferia do continente. As distribuições da $T_{a r}$ também são diferentes de estação para estação e as tendências podem ter sinais opostos mesmo em estações adjacentes (Jacka e Budd, 1991; Smith et al., 1996; Karl e Knight, 1994).

Nas últimas décadas, dados observados na Antártica têm mostrado que a $\mathrm{T}_{\mathrm{ar}}$ e pressão para níveis superiores mudaram significativamente. Uma tendência de aquecimento na Península Antártica e um pequeno resfriamento sobre o interior do continente Antártico são encontrados nas últimas décadas (Turner et al., 2005), e também um aquecimento significativo na Antártica Ocidental, atribuído às alterações regionais da circulação atmosférica (Steig et al., 2009).

O objetivo deste artigo é analisar a influência que os modos de variabilidade climática exercem sobre a variação da $\mathrm{T}_{\mathrm{ar}}$ na Antártica.

\section{MATERIAL E MÉTODOS}

\subsection{Dados}

Os dados de $\mathrm{T}_{\mathrm{ar}}$ do continente Antártico utilizados neste estudo foram obtidos através do Projeto READER - Reference Antarctic Data for Environmental Research (Turner et al., 2004), para o período de 1989 a 2009 . As estações estão localizadas conforme indicado na Figura 1. E foram obtidos por meio do site http://www.antarctic.ac.uk/met/READER/data. html, acessados em abril de 2011. O modo de variabilidade OA foi utilizado seguindo o critério de Marshall (2003), compreendendo o mesmo período dos dados de $\mathrm{T}_{\mathrm{ar}}$. Os dados mensais de ENOS foram obtidos por meio do NOAA no Earth System Research Laboratory na Physical Sciences Division.

Os dados de $\mathrm{T}_{\mathrm{ar}}$, os dados dos índices OA e ENOS foram trabalhados de forma semelhante. Os dados de $T_{a r}$ que no início eram horários ou em horário sinótico, passaram a ser mensais após a realização de média diária. Calculado a média mensal, foi procedida a média sazonal, dividindo em quatro períodos: verão (DJF), outono (MAM), inverno (JJA) e primavera $(\mathrm{SON})$

\subsection{Métodos de análise}

Com o objetivo de obter uma análise mais precisa de cada região do continente antártico, a região foi dividida em setores: Península Antártica e Shetlands do Sul (Bellingshausen, Ferraz e Rothera), Oceano Índico e Mar de Weddell (Halley, Neumayer e Novolazarevskaya), Oceano Pacífico (Davis, Mawson, Mirny e Casey), Mar de Ross (McMurdo, Marble Point, Ferrell e Gill) e Continental (Dome C e Amundsen Scott). 


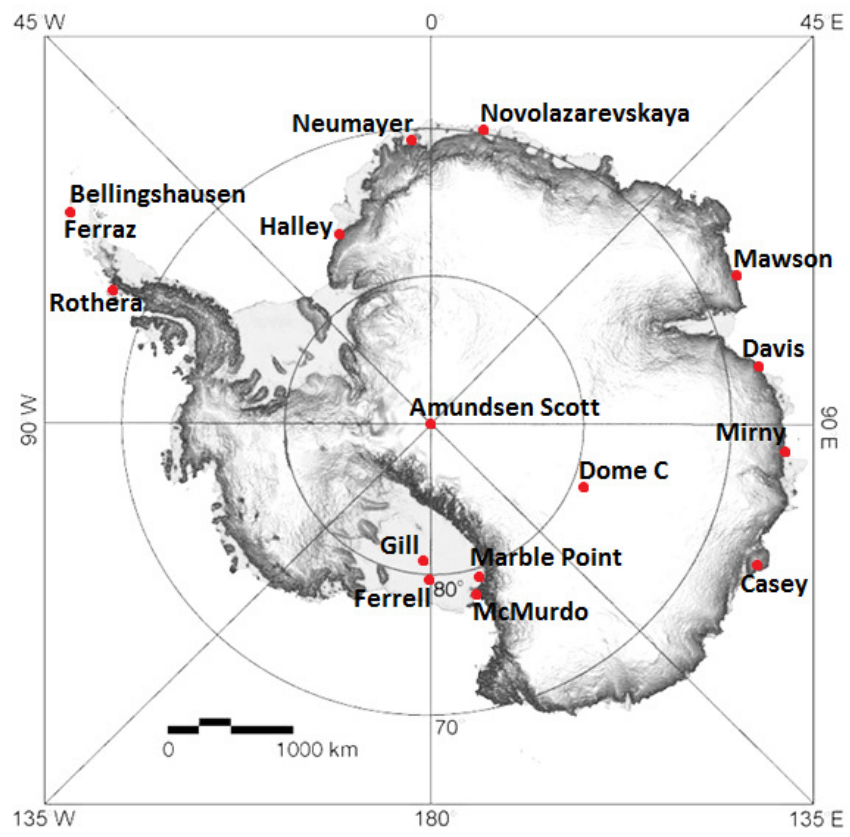

Figura 1 - Localização das estações automáticas.

Adaptado de "Antarctic Climate Change and the environment" Ano Polar Internacional 2007 - 2008.

Para o processamento estatístico das correlações foram realizadas médias sazonais a partir dos dados de $\mathrm{T}_{\mathrm{ar}}$, ENOS e OA. Assim, as correlações entre cada modo de variabilidade e a $\mathrm{T}_{\mathrm{ar}}$ serão analisadas para as estações (DJF, MAM, JJA e SON). A significância estatística será calculada com o teste t de Student.

\section{RESULTADOS E DISCUSSÃO}

Dado o grande número de estações meteorológicas somente as que apresentam os valores maiores e mais significativos em cada setor serão apresentadas.

É importante ressaltar que a correlação dos modos de variabilidade ENOS e OA com a $\mathrm{T}_{\text {ar }}$ para cada estação pode ser visualizada na Tabela 1 .

\subsection{Verão}

Os maiores valores de correlação encontrados entre a $\mathrm{T}_{\mathrm{ar}}$ e OA no verão para a região do oceano Pacífico, podem estar relacionados ao aumento dos ventos de oeste de média latitudes (Thompson et al., 2000) que podem favorecer a fase positiva da OA (ou seja, quanto mais baixas forem as pressões atmosféricas em altas latitudes, mais intensos serão os ventos de oeste). Então, as maiores correlações negativas entre a $\mathrm{T}_{\mathrm{ar}} \mathrm{e}$ a OA significam que na fase positiva da $\mathrm{OA}$ a $\mathrm{T}_{\text {ar }}$ na Antártica tendem a ser negativas.
A análise entre os dados observados e os modos de variabilidade climáticos mostra que o verão austral é caracterizado, principalmente, por correlações significativas entre a $\mathrm{T}_{\mathrm{ar}}$ das estações da Antártica Oriental e a OA, em particular as estações da região costeira do Oceano Pacífico. A estação Casey é significante em nível de 5\% $(-0,55)$, as outras estações são significantes a 1\% durante o verão, Davis, Mawson e Mirny apresentam as mais altas correlações negativas com a OA, -0,76, -0,80 e -0,69 respectivamente. Yu et al. (2011) e Marshall (2007) também encontraram resultados semelhantes com tendência forte de resfriamento para as estações de Mawson, Mirny, Casey e Amundsen Scott.

A Figura 2 representa uma correlação negativa entre $\mathrm{T}_{\mathrm{ar}}$ e OA, ou seja, OA com índice positivo coincide com anomalias negativas da $\mathrm{T}_{\mathrm{ar}}$. $\mathrm{O}$ verão austral é o único período em que Halley apresentou correlação significativa (10\% de significância), é o maior valor de correlação para a estação $(-0,40)$. As estações da região do Mar de Weddell e Oceano Índico apresentaram correlações negativas com bom índice de significância, por exemplo, Neumayer significativa a 10\% $(-0,49)$ e Novolazarevskaya significativa em nível de $1 \%$ $(-0,57)$ (Figura 2). A $T_{\text {ar }}$ das três estações desta região estão correlacionadas negativamente com a OA.

No setor continental, as estações são correlacionadas significativamente somente em nível de $10 \%$, por exemplo, Amundsen Scott (Figura 2) apresenta uma correlação de -0,43. 
Tabela 1 - Correlação entre OA e ENOS com a temperatura das estações localizadas no continente Antártico, para o período entre 1989-2009.

\begin{tabular}{|c|c|c|c|c|c|c|c|c|}
\hline \multirow{2}{*}{ Estações } & \multicolumn{2}{|c|}{ DJF } & \multicolumn{2}{|c|}{ MAM } & \multicolumn{2}{|c|}{ JJA } & \multicolumn{2}{|c|}{ SON } \\
\hline & OA & ENOS & OA & ENOS & OA & ENOS & OA & ENOS \\
\hline Bellingshausen & 0,29 & 0,13 & $0,65^{\star *}$ & $-0,30$ & $0,68^{* *}$ & $-0,25$ & $0,56^{* *}$ & $-0,44^{*}$ \\
\hline Ferraz & 0,27 & $-0,09$ & $0,71^{* *}$ & $-0,28$ & $0,70^{\star *}$ & 0,02 & $0,57^{*}$ & $-0,38$ \\
\hline Rothera & $-0,17$ & $-0,24$ & $0,58^{\star *}$ & $-0,37$ & $0,46^{*}$ & $-0,50^{*}$ & $0,53^{*}$ & $-0,50^{*}$ \\
\hline Halley & $-0,40$ & 0,21 & 0,22 & $-0,27$ & $-0,24$ & $-0,32$ & 0,25 & 0,07 \\
\hline Neumayer & $-0,49$ & 0,34 & $-0,54^{*}$ & 0,01 & $-0,11$ & 0,16 & $-0,10$ & 0,21 \\
\hline Novolazarevskaya & $-0,57^{* *}$ & $0,46^{*}$ & $-0,59^{\star *}$ & 0,36 & $-0,53^{*}$ & 0,03 & $-0,43^{*}$ & $-0,12$ \\
\hline Davis & $-0,76^{* *}$ & $0,45^{*}$ & $-0,49^{*}$ & $-0,26$ & $-0,48^{*}$ & $-0,19$ & $-0,62^{* *}$ & 0,02 \\
\hline Mawson & $-0,80^{\star *}$ & 0,34 & $-0,59$ ** & $-0,12$ & $-0,54^{*}$ & $-0,21$ & $-0,57^{\star *}$ & $-0,06$ \\
\hline Mirny & $-0,69^{\star *}$ & $0,49^{*}$ & $-0,57^{* *}$ & $-0,18$ & $-0,60^{\star *}$ & $-0,07$ & $-0,72^{\star *}$ & 0,06 \\
\hline Casey & $-0,55^{\star}$ & 0,32 & $-0,47^{*}$ & 0,05 & $-0,57^{\star *}$ & $-0,06$ & $-0,56^{*}$ & $-0,18$ \\
\hline McMurdo & $-0,08$ & 0,26 & $-0,51^{*}$ & 0,15 & $-0,19$ & 0,11 & 0,20 & 0,19 \\
\hline Marble Point & $-0,09$ & $-0,07$ & $-0,37$ & $-0,10$ & $-0,07$ & 0,19 & $-0,09$ & 0,39 \\
\hline Ferrell & $-0,19$ & $-0,15$ & $-0,32$ & $-0,34$ & $-0,03$ & $-0,38$ & $-0,33$ & 0,40 \\
\hline Gill & $-0,35$ & 0,38 & $-0,43$ & 0,13 & $-0,14$ & $-0,09$ & $-0,45$ & 0,43 \\
\hline Dome C & $-0,51$ & 0,19 & $-0,42$ & $-0,16$ & $-0,57^{\star *}$ & 0,09 & $-0,20$ & $-0,12$ \\
\hline Amundsen Scott & $-0,43$ & 0,20 & $-0,26$ & 0,03 & $-0,63^{* *}$ & $-0,28$ & $-0,18$ & 0,16 \\
\hline
\end{tabular}

Teste de Student $(\mathrm{t})=\mathbf{X}$ estatisticamente significativo a $10 \% ; \mathbf{X}^{*}$ significativo a $5 \% ; \mathbf{X}^{* *}$ significativo a $1 \%$.

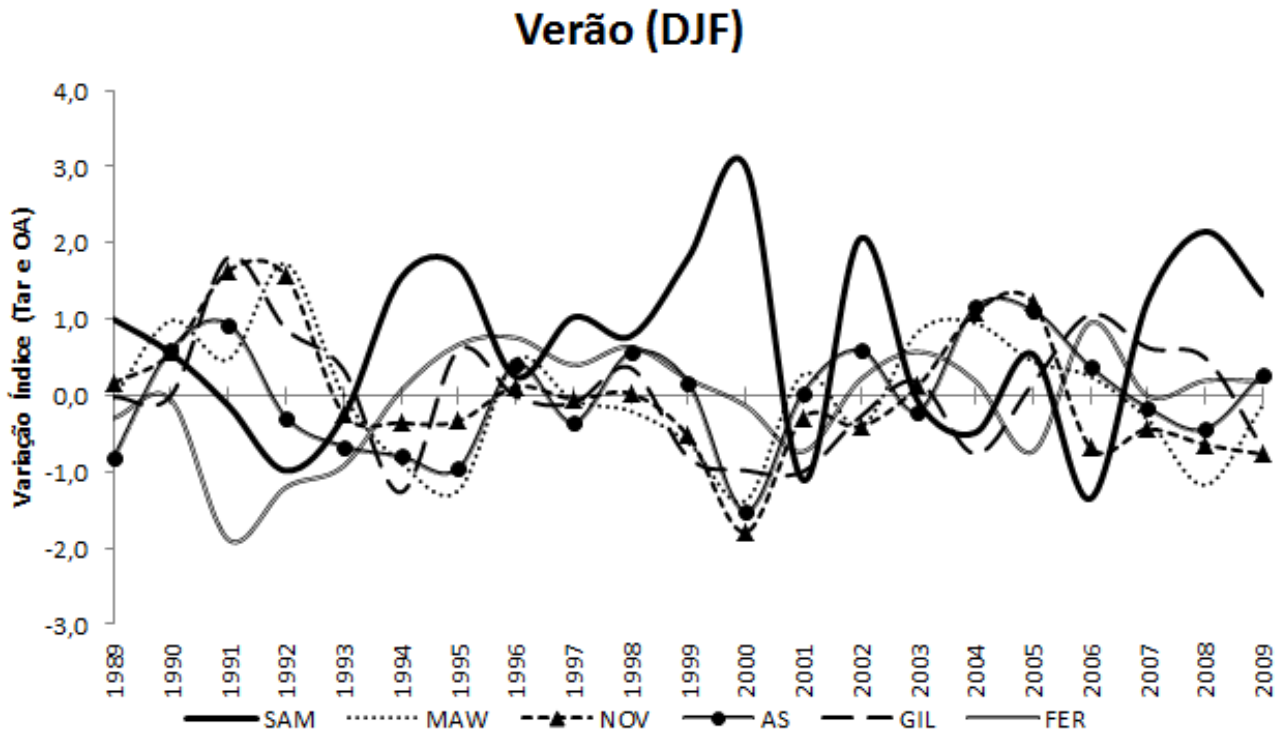

Figura 2 - Série temporal para o verão da OA (linha em negrito) e da temperatura do ar $\left({ }^{\circ} \mathrm{C}\right)$ em superfície nas estações meteorológicas: Mawson (MAW), Novolazarevskaya (NOV), Amundsen Scott (AS), Gill (GIL) e Ferraz (FER).

Nas regiões do Mar de Ross, Península Antártica e Shetland do Sul (Tabela 1) as correlações foram fracas, e não significativas entre OA e $\mathrm{T}_{\mathrm{ar}}$ das estações.

As avaliações entre a $\mathrm{T}_{\mathrm{ar}}$ e os eventos ENOS mostram que as estações de Novolazarevskaya $(0,46)$, Davis $(0,45)$ e Mirny $(0,49)$ (Figura 3), localizadas na região entre os oceanos Índico e Pacífico, apresentaram correlação positiva significativa a 5\%. No Mar de Ross, a estação Gill foi significativa a 10\% $(0,38)$ (Figura 3). Ainda de acordo com a Figura 3, na última década os eventos de El Niño (La Niña) tiveram menor (maior) ocorrência. De acordo com Carvalho et al. (2005) a convecção profunda no Pacífico central equatorial, o qual está relacionada com a La Niña (El Niño) que modula a circulação no HS e favorece a fase positiva (negativa) da OA durante o verão austral.

\subsection{Outono}

As correlações da OA com as $\mathrm{T}_{\mathrm{ar}}$ são positivas significativamente em nível de $1 \%$ para as três estações localizadas na Península Antártica e Shetland do Sul (Tabela 1), durante o período de outono austral (MAM): para Bellingshausen, o índice de correlação foi 0,65 (Figura 4). 


\section{Verão (DJF)}

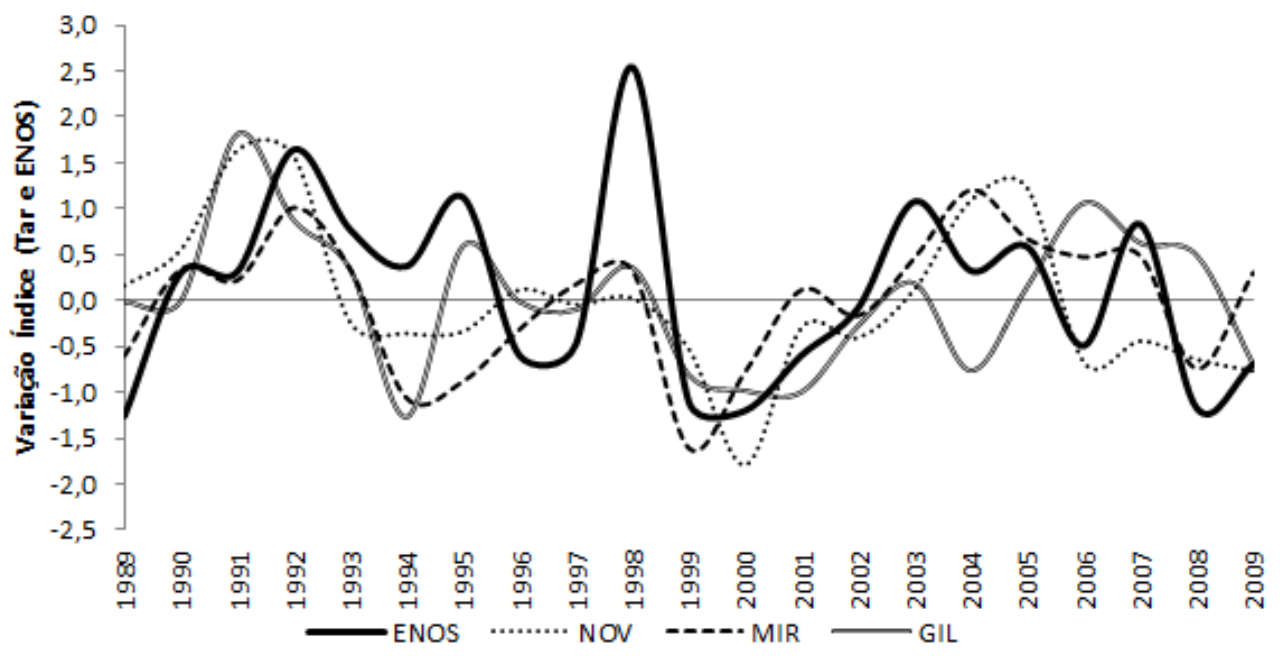

Figura 3 - Série temporal para o verão do ENOS (linha em negrito) e da temperatura do ar $\left({ }^{\circ} \mathrm{C}\right)$ em superfície nas estações meteorológicas: Novolazarevskaya (NOV), Mirny (MIR) e Gill (GIL).

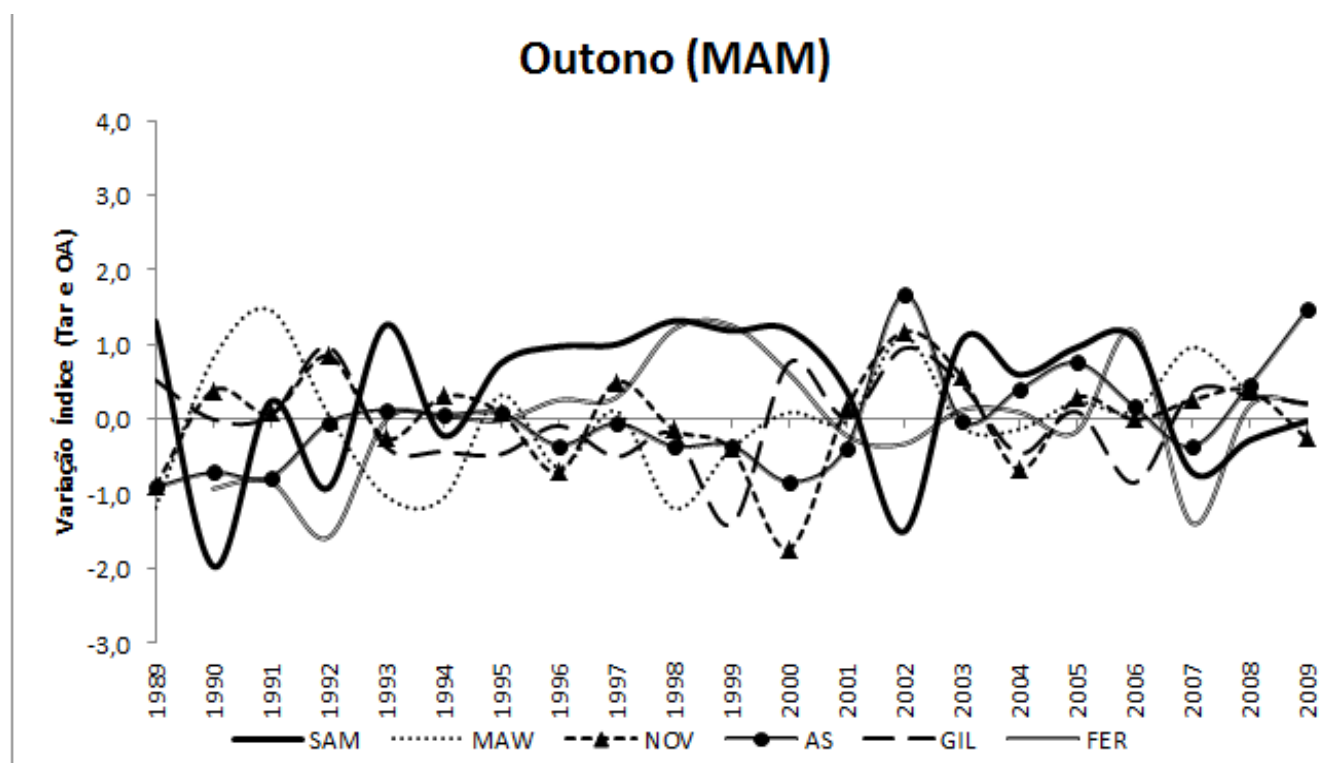

Figura 4 - Série temporal para o outono da OA (linha em negrito) e da temperatura do ar $\left({ }^{\circ} \mathrm{C}\right)$ em superfície nas estações meteorológicas: Mawson (MAW), Novolazarevskaya (NOV), Amundsen Scott (AS), Gill (GIL) e Ferraz (FER).

Ferraz obteve o maior índice com 0,71 e Rothera o menor $(0,58)$. Esse resultado é semelhante ao encontrado por Yu et al. (2011) e Steig et al. (2009).

Para as estações da região do Oceano Índico e Mar de Weddell a OA apresentou diferentes situações, enquanto para a estação de Novolazarevskaya a correlação foi negativa $(-0,59)$ e significativa estatisticamente em nível de 1\% (Figura 4), para estação de Neumayer a correlação foi significativa ao nível de 5\% $(-0,54)$. Assim como, encontrado por Marshall (2007), somente na estação de Halley a OA não foi significativa durante o outono.
Esta estação ainda apresentou a menor correlação encontrada entre todos os períodos do ano $(0,21)$ e diferentemente das outras duas estações com a correlação positiva.

Para as estações localizadas no setor oriental da Antártica, na região costeira do oceano Pacífico, foram encontradas correlações negativas entre a $\mathrm{OA}$ e a $\mathrm{T}_{\mathrm{ar}}$. Similar resultado foi encontrado por Thompson e Solomon (2002) e Yu et al. (2011). As estações Mawson (Figura 4) e Mirny foram significativas em nível de $1 \%$ e com índices de correlações de $-0,59$ e $-0,57$ respectivamente. As estações localizadas mais ao 
extremo da região, Davis $(-0,49)$ e Casey $(-0,47)$ apresentam correlações significativas em nível de 5\%. A estação Mawson juntamente com Novolazarevskaya apresentou o maior índice de correlação negativa entre todas as estações da Antártica para o período de outono austral.

A região do mar de Ross e o setor Continental da Antártica foram as regiões que apresentaram o menor índice de significância entre $\mathrm{OA}$ e a $\mathrm{T}_{\mathrm{ar}}$, para a primeira região citada, apenas a estação de McMurdo foi significativa em nível de 5\% (-0,51) (Figura 4). As estações Gill $(-0,43)$ e Marble Point $(-0,37)$ foram significativas a $10 \%$, assim como Dome C $(-0,42)$ na região continental (Figura 4). As estações Ferrell $(-0,32)$ e Amundsen Scott $(-0,26)$ não apresentaram correlações significativas durante o outono, embora mostre valores negativos.

Durante o outono austral, apenas uma estação apresentou correlação negativa significativa a 10\% com o ENOS, a estação Rothera $(-0,37)$ localizada na Península Antártica. Durante os 20 anos da série em estudo, a ocorrência de El Niño foi maior que os eventos de La Niña, porém na última década a intensidade dos El Niños foi menor. Isto poderia influenciar numa tendência menor de aquecimento da região, no entanto, a ocorrência da fase positiva da OA (como pode ser observado na Figura 4) contribui para a tendência de aquecimento na região (Yuan, 2004).

\subsection{Inverno}

O padrão espacial de correlações entre $\mathrm{OA}$ e $\mathrm{T}_{\mathrm{ar}}$ apresentada no inverno é semelhante ao outono, em geral as correlações são menores no inverno, e em alguns casos redução da significância das correlações. Das 16 estações em análise, apenas cinco estações apresentaram aumento no índice da correlação ou na sua significância estatística (Tabela 1). As estações de Bellingshausen (Figura 5) na região das Shetland do Sul $(0,68)$, e Mirny (Figura 5) na Antártica Oriental $(-0,60)$, foram as estações que aumentaram o seu índice de correlação e continuaram estatisticamente significantes em nível de $1 \%$.

É importante notar que na região da Antártica Oriental, Casey além de aumentar o seu índice de correlação entre a $\mathrm{OA}$ e $\mathrm{T}_{\mathrm{ar}}$, aumentou a sua significância estatística passando de $5 \%$ (encontrado no outono) para $1 \%$ durante o inverno $(-0,57)$. A região que merece maior destaque, todavia, é o setor continental. Este além de apresentar os seus maiores índices de correlações para o período, as duas estações foram significativas estatisticamente em nível de 1\%, Amundsen Scott com correlação negativa de -0,63 (Figura 5) e Dome C com -0,57.

Para as demais estações os valores de correlação ou a significância estatística foram menores se comparados aos valores encontrados durante o outono: Ferraz $(0,70)$, Davis $(-0,48)$ e Ferrell $(-0,03)$ apenas diminuíram o índice da correlação. As estações de Rothera $(0,46)$, Neumayer $(-0,11)$, Novolazarevskaya $(-0,53)$ (Figura 5), Mawson $(-0,54)$, McMurdo (-0,19) (Figura 5), Gill $(-0,14)$ e Marble Point $(-0,07)$ além de diminuir o índice da correlação a significância estatística também foi menor.

No inverno de modo geral, a menor correlação existente entre OA e a $\mathrm{T}_{\text {ar }}$, em relação ao outono, significa que as variações de $T_{\text {ar }}$ estão associadas a processos em escalas menores, como por exemplo, as ondas transientes (Marshall, 2007). A única

\section{Inverno (JJA)}

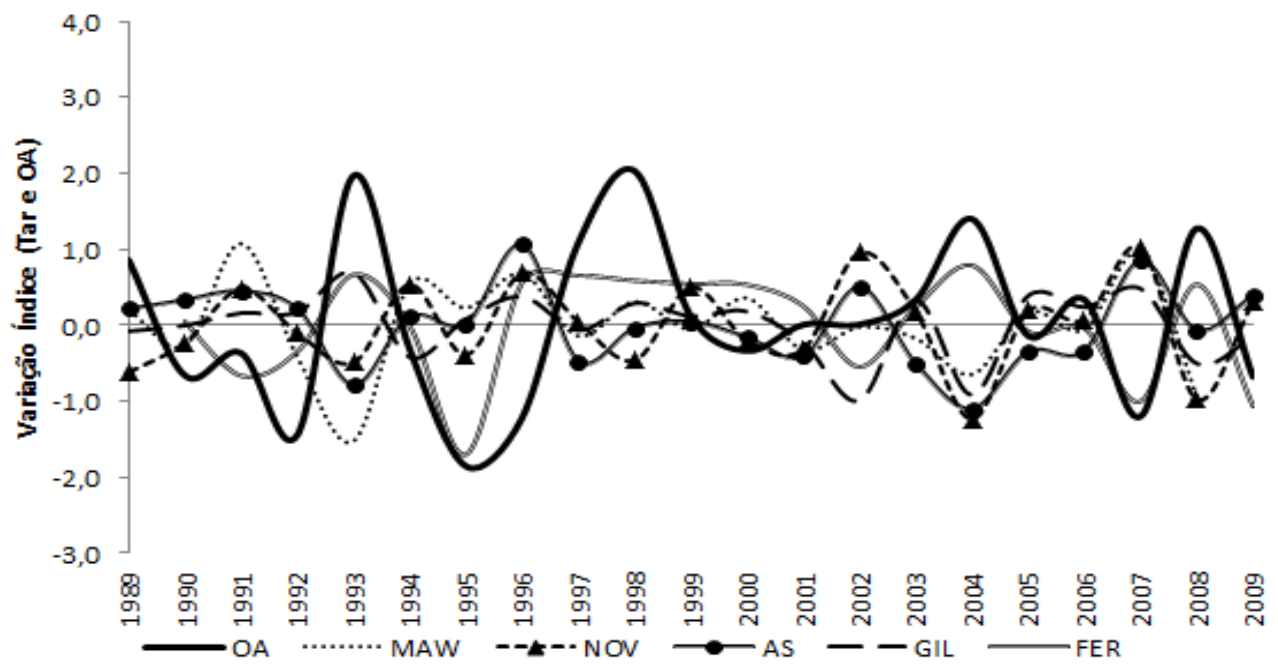

Figura 5 - Série temporal para o inverno da OA (linha em negrito) e da temperatura do ar $\left({ }^{\circ} \mathrm{C}\right)$ em superfície nas estações meteorológicas: Mawson (MAW), Novolazarevskaya (NOV), Amundsen Scott (AS), Gill (GIL) e Ferraz (FER). 
estação que apresentou resultado atípico das demais foi Halley, que durante o outono obteve baixa correlação positiva $(0,22)$ e passou para correlação negativa também fraca durante o inverno $(-0,24)$, esse resultado é contrário aos resultados encontrados nas demais estações e difere dos estudos obtidos por Marshall (2007). As razões que levam esta estação a esses resultados não estão bem esclarecidos pela literatura.

Assim como o outono, a única estação que obteve correlação significativa estatisticamente (em nível de 5\%) com o ENOS foi Rothera, (-0.50). Karoly (1989) observou trem de ondas durante o inverno austral em anos de El Niño, este trem de ondas afetam as condições sinóticas sobre a região sul da América do Sul e a Península Antártica. Deve-se notar que as respostas da $\mathrm{T}_{\mathrm{ar}}$ aos El Niños são mais intensas que aos eventos de La Niña (não apresentado) durante o inverno, isto, associado com a tendência positiva da OA (Figura 5), pode contribuir para o aquecimento na região oeste da Península Antártica, onde se localiza a estação Rothera.

\subsection{Primavera}

Durante a primavera as correlações existentes entre OA e a $\mathrm{T}_{\mathrm{ar}}$ não variam muito, para as estações em análise algumas considerações importantes devem ser, todavia, ressaltadas. É neste período que a correlação entre a $\mathrm{T}_{\mathrm{ar}}$ na Península Antártica e Shetland do Sul (Tabela 1) e OA diminui em relação ao outono e inverno. Para a estação Ferraz a significância diminui do nível de $1 \%(0,70)$ para $5 \%(0,57)$, assim como, para a estação de Novolazarevskaya $(-0,53$ para $-0,43)$ (Figura 6), na região do Oceano Índico e Casey (-0,57 para -0,56) no Oceano Pacífico.
Para as regiões do setor continental e do Mar de Ross (Figura 6), apenas a estação Gill é significativa a 10\% $(-0,45)$ e a estação McMurdo que inverte a sua correlação, passando de negativa $(-0,19)$ para positiva $(0,20)$ (única correlação positiva da estação para todo o período do ano). Essa diminuição no valor da correlação, também foi observado por Marshall (2007) durante o seu estudo para a estação de Vostok, que fica entre Amundsen Scott e Dome C. Na região da Antártica Oriental apenas Casey foi significante em nível de 5\% (-0,56), as demais estações foram significantes estatisticamente em nível de $1 \%$, o que demonstra a forte correlação existente entre a variação da $\mathrm{T}_{\mathrm{ar}}$ e a OA nesta região. Thompson e Wallace (2000) demonstraram que o período mais ativo da OA no HS é no final da primavera austral, e que a OA parece modular a intensidade da circulação média na baixa estratosfera, a altura da coluna de ozônio e a altura da tropopausa sobre latitudes médias e altas.

As estações da Península Antártica e Shetland do Sul apresentaram boa correlação com o ENOS. Bellingshausen e Rothera mostram correlações significativas estatisticamente a 5\% (-0,44 e -0,50), enquanto Ferraz a 10\% (-0,38) (Tabela 1). Outra região da Antártica que apresentou correlação entre $\mathrm{T}_{\mathrm{ar}}$ e ENOS foi o mar de Ross, com as estações Ferrell $(0,40)$, Gill $(0,43)$ (Figura 7) e Marble Point $(0,39)$ com significância de 10\%. De acordo com Fogt e Bromwich (2006), durante o período de primavera austral ocorre um aumento nos bloqueios atmosféricos sobre o Pacífico e aumento da pressão atmosférica em eventos de El Niño. Como pode ser observado na Figura 7, os eventos de El Niño foram em maior número que os eventos de La Niña durante o período de primavera austral. Durante o El Niño os ventos meridionais

\section{Primavera (SON)}

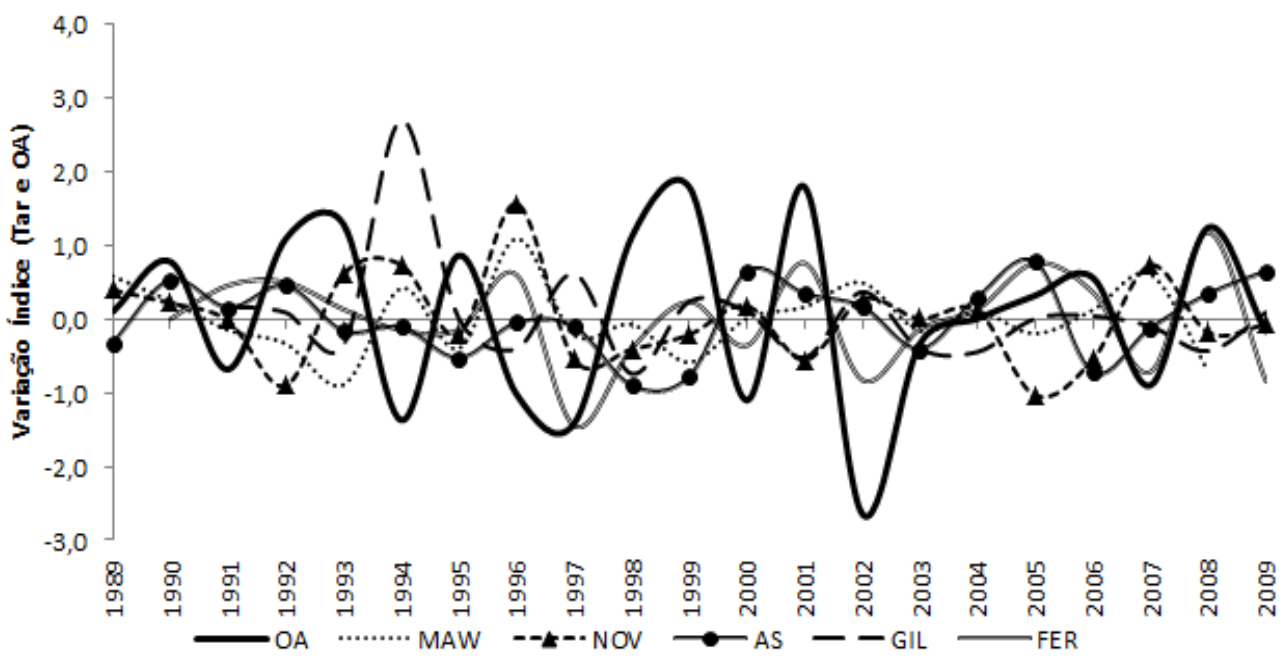

Figura 6 - Série temporal para a primavera da OA (linha em negrito) e da temperatura do $\operatorname{ar}\left({ }^{\circ} \mathrm{C}\right)$ em superfície nas estações meteorológicas: Mawson (MAW), Novolazarevskaya (NOV), Amundsen Scott (AS), Gill (GIL) e Ferraz (FER). 


\section{Primavera (SON)}

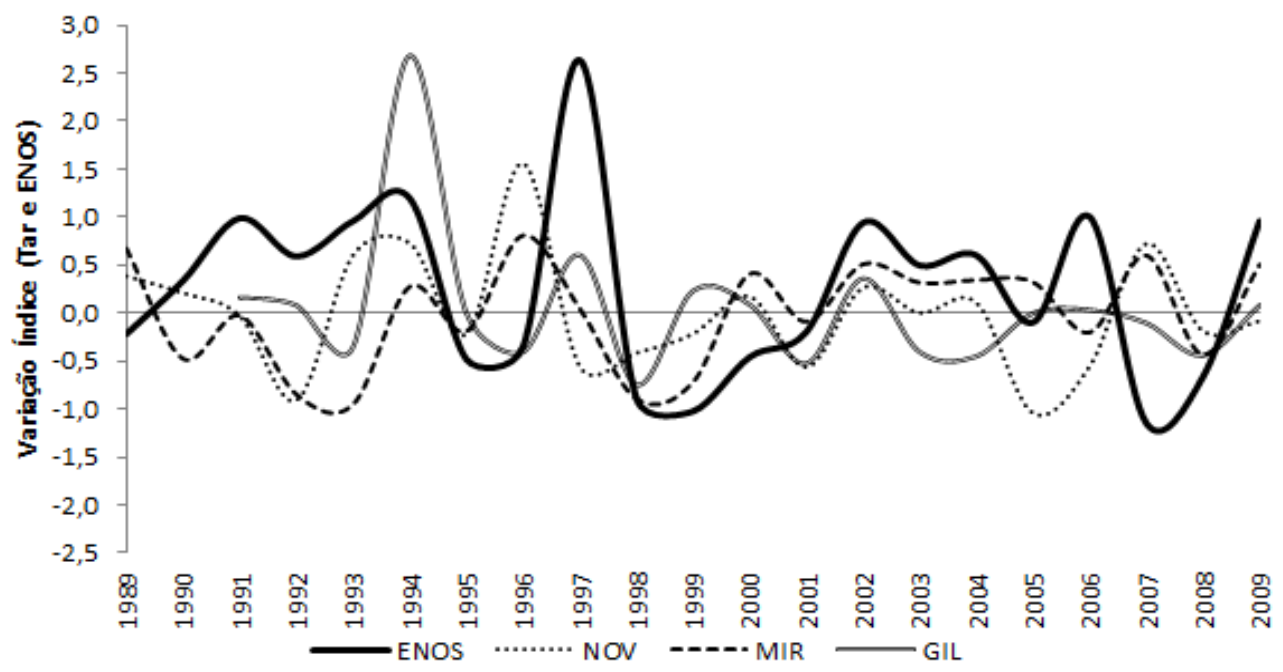

Figura 7 - Série temporal para a primavera do ENOS (linha em negrito) e da temperatura do ar $\left({ }^{\circ} \mathrm{C}\right)$ em superfície nas estações meteorológicas: Novolazarevskaya (NOV), Mirny (MIR) e Gill (GIL).

são intensificados na região da Península transportando calor proveniente de latitudes mais baixas. No setor do Mar de Ross a tendência é de aquecimento devido ao El Niño, o que é contrário ao observado na Península Antártica. Os eventos de El Niño, apesar de serem em maior frequência, diminuíram de intensidade na última década se comparado com a década de 1980. Isto pode ser uma das causas para a tendência de aquecimento na região durante a primavera.

\section{CONCLUSÕES}

Durante o verão austral, a OA apresentou significativa contribuição para a variação da $T_{a r}$, principalmente para as estações dos setores do Mar de Weddell, Oceano Índico e setor oriental da Antártica, para a última região citada as correlações negativas foram estatisticamente significantes. No setor continental apesar da pequena tendência de aquecimento, a OA apresentou correlações negativas e significantes estatisticamente de acordo com o teste $t$ de Student. Nas regiões do Mar de Ross, Península Antártica e Shetland do Sul não foi observada variações significativas associadas com a $\mathrm{OA}$.

O presente trabalho utilizou uma série histórica mais recente que a maioria da literatura, e mesmo assim os resultados encontrados neste trabalho são condizentes com os resultados encontrados em outras pesquisas semelhantes, com uma forte correlação entre a $\mathrm{OA}$ e o comportamento da $\mathrm{T}_{\mathrm{ar}}$ na Antártica durante todas as estações do ano, apresentando um comportamento de dipolo entre os setores da Península Antártica e o setor oeste da Antártica (setores dos oceanos Pacífico e Índico).
É importante notar a influência do ENOS na região oeste da Península Antártica, principalmente durante a primavera, com correlações negativas significantes entre o modo de variabilidade e a $\mathrm{T}_{\mathrm{ar}}$.

\section{AGRADECIMENTOS}

Ao $\mathrm{CNPq}$ pelo apoio financeiro durante a pesquisa. Ao READER pela disponibilidade dos dados observados de temperatura do ar em superfície.

\section{REFERÊNCIAS BIBLIOGRÁFICAS}

CARVALHO, L.M.V.; JONES, C.; AMBRIZZI, T. Opposite phases of the Antarctic Oscillation and relationships with intraseasonal to interanual activity in the tropics during the austral summer, Journal of Climate, v. 18, p. 702-718, 2005.

FELDSTEIN, S.B. Teleconnections and ENSO: the timescale, power spectra, and climate noise properties. Journal of Climate, v. 13, p. 4430-4440, 2000.

FOGT, R.L.; BROMWICH, D.H. Decadal variability of the ENSO teleconnection to the high-latitude south Pacific governed by coupling with the southern annular mode. Journal of Climate, v. 19, p. 979-997, 2006.

HOSKINS, B.J.; KAROLY, D.J. The steady linear response of a spherical atmosphere to thermal and orographic forcing. Journal of the Atmospheric Sciences, v. 38, p. 1179-1196, 1981.

JACKA, T.H.; BUDD, W.F. Detection of temperature and sea ice extent changes in the Antarctic and Southern Ocean. 
Weller, G., Wilson, C. L. and Severin, B. A. Proceedings of the International Conference on the Role of the Polar Regions in Global Change. June 11 - 15 1990, University of Alaska Fairbanks, 63 - 70. Fairbanks, AK, University of Alaska, Geophysical Institute, 1991.

JACKA, T.H.; BUDD, W.F. Detection of temperature and seaice-extent changes in the Antarctic and Southern Ocean, 1949 - 1996. Annals of Glaciology, v.27, p. 553 - 559, 1998.

JUSTINO, F.B.; PELTIER, R. Climate anomalies induced by the Arctic and Antarctics Oscillations: Glacial Maximum and presente-day perspectives. Journal of Climate, v. 21, n. 3 p. 459-475, 2008.

KARL, T.R.; KNIGHT, R.W. Global and hemispheric temperature trends: Uncertainties related to inadequate spatial sampling. Journal of Climate, v. 7, p. $1144-1163$, 1994.

KAROLY, D.J. Southern Hemisphere circulation features associated with El Niño-Southern oscillation events. Journal of Climate, v. 2, p. 1239-1252, 1989.

KING, J.C.; TURNER, J. Antarctic meteorology and climatology. Cambridge: Cambridge University Press, 409p., 1997.

MARSHALL, G.J. Trends in the southern annular mode from observations and reanalyses. Journal of Climate, v. 16, p. 4134-4143, 2003.

MARSHALL, G.J. Short communication: half-century seasonal relationships between the Southern Annular Mode and Antarctic temperatures. International Journal of Climatology, v. 27, p. 373-383, 2007.

MO, K.C.; WHITE, G.H. Teleconnections in the Southern Hemisphere. Monthly Weather Review, v. 113, p. 22-37, 1985.

RAPER, S.C.; WIGLEY, T.M.; JONES, P.D.; SALINGER, M.J. Variations in surface air temperatures: part 3. The Antarctic, 1957 - 1982. Monthly Weather Review, v. 112, p. 1341 $-1353,1984$.
SMITH, R.C.; STAMMERJOHN, S.E.; BAKER, K.S. Surface air temperature variations in the Western Antarctic Peninsula Region. Foudations for Ecological Research West of Antarctic Peninsula, R. M. Ross et al., Eds., Antarctic Research Series, v. 70, p. $104-121,1996$.

STEIG, E.J.; SCHNEIDER, D.P.; RUTHERFORD, S.D. et al. Warming of the Antarctic ice-sheet surface since the 1957 International Geophysical Year. Nature, v. 457, p. 459-463, 2009.

THOMPSON, D.W.J.; SOLOMON, S. Interpretation of recent Southern Hemisphere climate change. Science, v. 296, p. 895-899, 2002.

THOMPSON, D.W.J.; WALLACE, J.M. Annular modes in the extratropical circulation: Part I: Month-to-month variability. Journal of Climate, v. 13, n. 5, p. 1000-1016, 2000.

THOMPSON, D.W.J.; WALLACE, J.M.; HEGERL, G.C. Annular modes in the extratropical circulation: trends. Journal of Climate, v. 13, p. 1018-1036, 2000.

TURNER, J.; COLWELL, S.R.; MARSHALL, G.J. et al. The SCAR READER project: towards a high-quality database of mean Antarctic meteorological observations. Journal of Climate, v. 17, p. 2890-2898, 2004.

TURNER, J.; COLWELL, S.R.; MARSHALL, G.J. et al. Antarctic climate change during the last 50 years. International Journal of Climatology, v. 25, p. 279-294, 2005.

YU, L.; ZHANG, Z.; ZHOU, M. et al. Influence of the Antarctic oscillation, the Pacific-South American modes and the El Niño-Southern oscillation on the Antarctic surface temperature and pressure variations. Antarctic Science, p. 1-18, 2011.

YUAN, X. ENSO-related impacts on Antarctic sea ice: a synthesis of phenomenon and mechanisms. Antarctic Science, v. 16, p. 415-425, 2004. 